guten Einblick in verschiedene Bereiche, aktuelle Projekte und Initiativen sowie zahlreiche Anknüpfungspunkte für die weitere Auseinandersetzung mit der Thematik.

Claudia Lampert

\section{Sabine Krajewski}

\section{Life goes on, and sometimes it doesn't}

A comparative study of medical drama in the US, Great Britain and Germany

Frankfurt: Lang, 2002. - $220 \mathrm{~S}$.

(Europäische Hochschulschriften, Reihe 30; 85)

(Zugl.: Frankfurt (Oder), Europa-Univ., Diss., 2001)

ISBN 3-631-39824-7

Die Umstände, die zur vorliegenden Studie geführt haben, hatten, so die Verfasserin, zunächst wenig mit wissenschaftlichem Interesse $\mathrm{zu}$ tun. Ausschlaggebend war eher die Beobachtung, dass im britischen und amerikanischen wie auch im deutschen Fernsehen ausgangs des letzten Jahrtausends kaum ein Tag verging, an dem man nicht mindestens einmal am Tag auf eine Krankenhausserie stieß. Auch wenn sich die Fülle an Arzt- und Krankenhausserien nunmehr reduziert hat, so steht der Donnerstag Abend auf Sat.1 immer noch Pate für den stabilen Erfolg der Serien, der die Relevanz einer Auseinandersetzung mit dem Genre verdeutlicht. Ein Blick auf die verschiedenen Gesundheitssysteme in Deutschland, Großbritannien und USA wirft zudem die Frage auf, ob der Erfolg der Serien nicht auf die Probleme und Veränderungen im Gesundheitswesen zurückzuführen ist: In Großbritannien waren vor allem in den 80er Jahren gravierende Einschnitte zu beobachten. Dies geschah dann im deutschen System der 90er, während das Gesundheitssystem in den USA schon immer mit Problemen zu kämpfen hatte.

Ausgehend von dieser Beobachtung will die Verfasserin der vorliegenden Studie nicht nur die kulturellen Unterschiede zwischen deutschen, britischen und amerikanischen Krankenhausserien aufzeigen, sondern auch untersuchen, inwieweit der Erfolg der Serien mit der Entwicklung des Gesundheitswesens in den drei Ländern einhergeht. Zentrales Untersuchungsziel ist dabei, Unterschiede zwischen den Serien der drei verschiedenen Länder zu identifizieren, um auf dieser Basis kulturelle Unterschiede zu identifizieren und so Material $\mathrm{zu}$ generieren, das im Sprachunterricht im Kontext interkultureller Kommunikation genutzt werden kann.

Methodisch stützt sich Krajewski dabei vor dem Hintergrund der britischen Cultural-Studies-Forschung auf die qualitative Inhaltsanalyse und kritische Diskursanalyse. Daher dient dieser Ansatz auch als Ausgangspunkt für die theoretischen Ausführungen, die in einer kritischen Auseinandersetzung mit qualitativen und quantitativen Methoden generell und der angewandten Methodik münden. Im zweiten Teil befasst sich die Verfasserin mit dem Genre der Krankenhausserie und dessen Entwicklung, um sich daraufhin im empirischen Teil der Studie mit den kulturellen Unterschieden der Krankenhausserien diskursanalytisch auseinander zu setzen.

Für einen Kommunikationsforscher, der sich hauptsächlich im Mainstream klassischer quantitativer Forschung verankert sieht, ist die Studie nicht nur deshalb wertvoll, weil sie die Perspektive der Cultural Studies genauso wie die Methode der kritischen Diskursanalyse ausführlich und nachvollziehbar vorstellt und ihren Wert im Kontext der vorliegenden Fragestellung evident macht, sondern auch, weil sie auf Theorien zum Zusammenhang von Massenmedien und Massenkultur eingeht, auf die wir nicht täglich stoßen; so z. B. die „Triple M Theory", die das Dreiecksverhältnis zwischen Medien, Gesellschaft und Kultur beschreibt und sich damit auseinander setzt, wie Massenmedien Bedeutung und Werte determinieren. Cultural Studies werden dabei definiert als kritisch-interpretativer Ansatz, der die tägliche Produktion von Kultur analysiert und seinen Fokus auf Mechanismen sozialer Beziehungen und sozialer Ordnung legt, durch die Kultur erzeugt und wahrgenommen wird. Inhärent ist dabei die Idee, dass massenmediale Produkte, kontrolliert von international operierenden Konzernen, homogene Muster sozialen Verhaltens beeinflussen oder sogar erzeugen. In diesen Kontext ordnet sich auch die Frage nach einer Amerikanisierung von Krankenhausserien ein: Die Dominanz US-amerikanischer Produktionen, die europäischen Krankenhausserien häufig als Vorbild dienen, deutet diese Tendenz an, der vergleichsweise geringe Erfolg der original-amerikanischen Krankenhausse- 
rien in europäischen Ländern spricht allerdings eher für die Macht der eigenen Kultur.

Vor dem Hintergrund dieser Vorüberlegungen steht der kulturelle Vergleich der Krankenhausserien im Zentrum des Forschungsinteresses. Hierfür setzt sich die Studie zum einen mit den Produktionsprozessen auseinander (Gespräche mit Produzenten), zum anderen mit der internen Struktur der Serien. Ziel ist die Identifikation von Repräsentationsmustern im Hinblick auf die dargestellten Charaktere, Machtstrukturen und Handlungen, die auf zwei Achsen von Bedeutung sind: Die Entwicklung der Serien über die Zeit hinweg (80er Jahre versus 90er) sowie der interkulturelle Vergleich amerikanischer, britischer und deutscher Serien. Einhergehend mit der kritischen Diskursanalyse wird dabei nicht nur gefragt, wie etwas dargestellt wird, sondern auch, warum es so und nicht anders geschieht, um auf diese Weise Implikationen für die soziale Struktur und kulturelle Identität sowie ihre politische Bedeutung zu identifizieren. Eine quantitative Vorgehensweise würde nicht ausreichen, dies zu klären, weil, so die Verfasserin, ein Medienprodukt wie das der Krankenhausserie viele Elemente enthält, die nicht gezählt werden könnten (z. B. visuelle Elemente, nonverbale Kommunikation oder Bezüge zu andere Texten). Zwar können quantitative Inhaltsanalysen Häufigkeiten, Tendenzen, Intensitäten und Zusammenhänge identifizieren, doch der Komplexität der Frage, wie Zuschauer die Weltsicht wahrnehmen, können sie nicht gerecht werden, weshalb sich die Verfasserin für die qualitative Vorgehensweise entscheidet. Zwar wird an der kritischen Diskursanalyse häufig bemängelt, dass sie einer ideologischen Interpretation gleicht, deren Ergebnisse offen sind für Diskussion und Wandel, doch können gerade diese Aspekte auch für diese Methode sprechen. Untersuchungsmaterial bilden jeweils sechs aufeinander folgende Episoden von zwei amerikanischen (Emergency Room, Chicago Hope), zwei britischen (Casuality, Holby City) und vier deutschen Krankenhausserien (Schwarzwaldklinik, Stadtklinik, Alphateam, OP ruft Dr. Bruckner). Mayring's Schritten der interpretativen Analyse folgend reduziert die Verfasserin das Material hierfür zunächst auf die relevanten Inhalte, strukturiert diese in Kategorien, um bedeutsame Muster zu identifizieren und diese wiederum anhand des Materials zu extrahieren, zu analysieren und interpretieren.
Nach einer Vorstellung des Genres der Krankenhausserie im zweiten Kapitel stellt die Verfasserin die so generierten Ergebnisse im dritten Abschnitt vor. Die untersuchten Sendungen werden dabei zunächst im Hinblick auf die Anzahl gezeigter Patienten quantitativ beschrieben, um hierauf diskursanalytisch Gemeinsamkeiten und Unterschiede aufzudecken. Diese werden expliziert auf Basis ausgewählter Aspekte, wie enthaltender Sozialkritik, dargestellter Kritik am Gesundheitswesen, Wertesystem, Machtstrukturen (Beziehungen innerhalb des Krankenhauspersonals, zwischen medizinischem Personal und Patienten), Tabuthemen (explizite, implizite, vermiedene Tabus) und schließlich humorspezifische Unterschiede.

Eine Zusammenfassung der Befunde zu liefern, würde den Rahmen der Rezension sprengen und wäre nur schwer zu gewährleisten was nicht zuletzt die Tatsache zeigt, dass die Verfasserin selbst mit Zusammenfassungen und Zwischenfazits äußerst sparsam umgeht. Es bleibt mehr dem Leser überlassen, sich in dem Sammelsurium an Interpretationen zurecht zu finden. So wirft die Verfasserin mit ihrem Buch zwar sehr interessante Fragen auf sowohl im Hinblick auf den interkulturellen Vergleich als auch bezüglich des Zusammenhangs zwischen Erfolg der Krankenhausserien und den Entwicklungen des Gesundheitswesens in der Realität. Was dies alles bedeutet, muss sich der Leser jedoch selbst zurecht suchen. Wenn man sich allerdings dieser Mühe unterzieht, eröffnet die qualitative Vorgehensweise hier Sichtweisen, die der quantitativen Forschung Grenzen aufzeigt. Beide Vorgehensweisen können sich also gegenseitig befruchten. Insgesamt hinterlässt die Studie gewollt oder nicht - zum Schluss ein großes Fragezeichen, weil sie ihre Qualitäten nicht zu nutzen vermag, sondern doch nur einer Aneinanderreihung einzelner Sequenzen verhaften bleibt, ohne zum Schluss generell etwas über die Serien sagen zu können. Damit ist nicht gemeint, dass wir aus der vorliegenden Stichprobe nicht auf alle Krankenhausserien schließen können - wie es die Verfasserin ja selbst konstatiert und gar nicht verlangt -, sondern dass schlicht ein weiterer Abstraktionsschritt fehlt, der das Potenzial des umfassenden, gewissenhaft und detailliert analysierten Materials nutzt.

Als Fazit erfahren wir, dass ein starker Einfluss amerikanischer Serien auf das europäische Fernsehen zu beobachten ist, dass die nationa- 
len Produkte „zu Hause“ aber deshalb erfolgreicher sind, weil die Art und Weise der Behandlung bestimmter Themen sehr unterschiedlich ist. So vertreten die Krankenhausserien aus allen drei Ländern bestimmte Werte wie Teamarbeit und Aufrichtigkeit, aber sie stellen diese Werte unterschiedlich dar, was am Beispiel dargestellter Hierarchien, des Humors und auch politischer Anspielungen besonders deutlich wird. Die ursprüngliche Annahme, dass es zwischen den Entwicklungen im Gesundheitswesen und der Entwicklung von Krankenhausserien eine Verbindung gibt, zeigte sich nicht. Doch stellen die Befunde eine gute Basis dar, über gesellschaftliche Entwicklungen und den Bedarf nach gesellschaftlichen Veränderungen in verschiedenen Kulturen nachzudenken.

Constanze Rossmann

\section{Otfried Jarren / Hartmut Weßler (Hrsg.)}

Journalismus - Medien - Öffentlichkeit

Eine Einführung

Opladen: Westdeutscher Verlag, 2002. - 413 S. ISBN 3-531-13514-7

Der Band „Journalismus - Medien - Öffentlichkeit" ist als Einführungswerk konzipiert. Während es zahlreiche Einführungen in die Kommunikationswissenschaft gibt, die die Bereiche „Journalismus" und „Öffentlichkeit“ mitbehandeln, fehlt es an klassischen Einführungstexten, zumal an solchen, die keinen Schwerpunkt auf die journalistische Praxis legen und die keiner bestimmten theoretischen Ausrichtung verpflichtet sind. Das Erscheinen dieses Bandes, der sich an „berufstätige und angehende Journalistinnen und Journalisten sowie [an] Studierende und Lehrende in kommunikationswissenschaftlichen Studiengängen“" richtet, ist daher grundsätzlich zu begrüßen.

Positiv hervorzuheben ist dabei vor allem die Zielsetzung des Buches, dem es darum geht, dem Leser zu ermöglichen, „(...) den Paradigmenstreit $\mathrm{zu}$ verstehen und sich ein Urteil $\mathrm{zu}$ bilden, nicht darum, die letztverbindliche Lehrmeinung ,aufzusaugen'“ (S. 25). Dieses Ziel wird cum grano salis durchgängig umgesetzt; dem mit den gängigen Theorien (noch) nicht vertrauten Leser wird also weder, wie in manchen anderen Einführungsbänden, mehr oder weniger offen eine theoretische Lesart „untergeschoben“, noch stehen verschiedene theoretische Ansätze unverbunden nebeneinander. Angenehm ist auch die Zurückhaltung aller Autoren hinsichtlich mancher „Modeerscheinungen" des Fachs: Barbara Pfetsch und Stefan Wehmeier etwa verzichten in ihrem Beitrag darauf, die ,inflationäre Zahl an Spezialbegriffen “ (S. 72) zu erläutern, die sich im Bereich Public Relations - gelegentlich ohne dass eine analoge Ausdifferenzierung der zu bezeichnenden Sachverhalte erkennbar wäre - herausgebildet haben.

Positiv hervorzuheben ist auch die für Herausgeberbände eher unübliche „Einigung“ auf ein allen Beiträgen zugrunde liegendes ,vereinfachtes Modell öffentlicher Kommunikation" (S. 29) - eine durchaus gelungene Mischung zwischen einem kommunikationstheoretischen Modell und einer visualisierten Buchgliederung, die in jedem Fall der Orientierung dient. Hier ist der Band tatsächlich im besten Sinne einfach, ohne zu stark zu vereinfachen. Die einzelnen Elemente des Modells, nämlich "Gesellschaftliche Akteure" (Barbara Pfetsch/ Stefan Wehmeier: „Sprecher: Kommunikationsleistungen gesellschaftlicher Akteure"), „Medien als institutioneller Kontext" (Otfried Jarren/Werner A. Meier: „Mediensysteme und Medienorganisationen als Rahmenbedingungen für den Journalismus“), „Journalisten als Rollenträger" (Frank Esser/Hartmut Weßler: "Journalisten als Rollenträger"), „Medienprodukte als Handlungsergebnisse" (Ralph Weiß: „Publizistische Medienprodukte - im Blick der Kommunikationswissenschaft") und „Publikum“ (Uwe Hasebrink: „Publikum, Mediennutzung und Medienwirkung") werden in separaten Kapiteln behandelt, die jeweils mit einer optisch hervorgehobenen Zusammenfassung abschließen.

Der Wunsch der Herausgeber, dem Leser Orientierungshilfen etwa in Gestalt solcher Zusammenfassungen $\mathrm{zu}$ geben, ist durchaus begrüßenswert. Dies hat jedoch auch zu einer komplexen Bildsymbolik in Form sechs verschiedener Piktogrammtypen geführt, die jeweils an den Seitenrändern auf bestimmte Textelemente verweisen. Dabei wird etwa auch auf Abbildungen und Tabellen mit einem Piktogramm verwiesen, wenngleich die Abbildungen durchaus auch ohne das Piktogramm mehr als deutlich erkennbar wären. Solcherlei Redundanz ist unnötig und verstärkt nur den Eindruck eines ohnehin recht unruhigen Layouts. Für den auf visuelle Orientierung ausgerichte- 